\title{
Some Mathematical Formulations of Electrochemical Polymerization Kinetics
}

\author{
S. N. BhadANi, R. S. Prasad, and G. PARRAVANO**** \\ Department of Chemistry, Ranchi University, Ranchi 834008, India. \\ * Department of Chemical Engineering, University of Michigan, Ann Arbor, Michigan 48109, U.S.A.
}

(Received December 23, 1981)

\begin{abstract}
Some theoretical aspects of electrochemical polymerization kinetics are discussed in this paper. Mathematical relationships between electrolysis current and the initial rate of polymerization are derived. Also, emphasis is placed on the derivation of mathematical relationships between electrolysis current and the kinetic chain length of polymers. From the Tafel relationship of current and electrode potential, various limiting mechanistic steps in the kinetics of electrochemical initiation by direct electron transfer between monomer and electrode are also illustrated.
\end{abstract}

KEY WORDS Electrochemical Polymerization Kinetics / Kinetic Chain Length / Electrode Potential / Tafel Relationship / Diffusion /

Electrochemical polymerization (ECP) is a recent branch of electrochemistry on which, within the last few years, several reviews have appeared in the literature. ${ }^{1-4}$ The typical features of ECP emerge from a combination of the principles of electrochemistry with those of polymer science. ${ }^{5}$

The goal of kinetic analysis of ECP is to understand the various reaction steps (such as electron transfer between electrodes and monomers followed by dimerization of radical-ions), their nature, rate and temporal sequence. The overall cell reaction may be separated into (1) two electrode reactions and (2) bulk solution reactions. In order to characterize the electrode kinetics, it is necessary to obtain experimental relationships between (a) the independent variables: current intensity, electrode potential, concentration of reactants, temperature, cell geometry, etc., and (b) the dependent variables: rate of polymer formation and reactant consumptions, degree of polymerization, molecular weight distribution, etc. In addition, the following quantities may be determined: electron transfer coefficients, reaction order, limiting diffusion, surption, reaction currents, stoichiometric factor, and overpotentials. A detailed discussion of the influence of these

\footnotetext{
** Deceased.
}

factors on electrode kinetics may be found elsewhere. ${ }^{6}$ However, unlike conventional polymerization, little theoretical work has been done on electrochemical polymerization. ${ }^{4,5}$ Thus, the present article describes some mathematical formulations of the kinetics of ECP. Emphasis is placed on the electron transfer steps involved in the initiation of polymerization at electrodes and the influences of chemical reactions on the electrode processes.

\section{RESULTS AND DISCUSSION}

\section{General Consideration on ECP Rates}

A typical ECP system consists of an electrolytic cell with electrodes, electrolytes, monomer, and solvent. The solvent is generally needed whenever the monomer is not soluble in the electrolyte. When an electrical current flows through the system, electrode reactions take place.

We shall assume that monomer molecules and electrons are efficiently utilized to produce a polymer. That is, all the reacted monomer becomes a part of the final polymer produced, and all electrons transferred between electrode and solution initiate the formation of at least one polymer chain. The rate of monomer consumption is therefore 


$$
-\frac{\mathrm{d}[\mathrm{M}]}{\mathrm{d} t}=k_{\mathrm{i}}[\mathrm{M}][\mathrm{I}]+k_{\mathrm{p}}[\mathrm{M}][\mathrm{P} *]+k_{\mathrm{trm}}[\mathrm{M}][\mathrm{P} *]
$$

where

$$
k_{\mathrm{i}}=\text { initiation constant, }
$$

$k_{\mathrm{p}}=$ propagation constant,

$k_{\text {trm }}=$ transfer constant to monomer,

$[\mathrm{M}]=$ monomer concentration,

$[\mathrm{I}]=$ initiator concentration,

and

$\left[\mathrm{P}^{*}\right]=$ active polymer-growing site.

It is likely that all terms in eq 1 are involved in many ECP systems under certain conditions. However, it is reasonable (and desirable) to expect that the ECP conditions may be regulated for any particular system so as to eliminate one or more of the terms in eq 1 .

The rate of chain growth is

$$
R_{\mathrm{p}}=k_{\mathrm{p}}[\mathrm{M}]\left[\mathrm{P}^{*}\right]
$$

and the rates of initiation, $R_{\mathrm{i}}$, termination, $R_{\mathrm{t}}$, and transfer, $R_{\mathrm{tr}}$, are

$$
\begin{aligned}
& R_{\mathrm{i}}=k_{\mathrm{i}}[\mathrm{M}][\mathrm{I}] \\
& R_{\mathrm{t}}=k_{\mathrm{tc}}\left[\mathrm{P}^{*}\right]^{2}+k_{\mathrm{td}}\left[\mathrm{P}^{*}\right]^{2} \\
& R_{\mathrm{tr}}=k_{\mathrm{trm}}[\mathrm{M}]\left[\mathrm{P}^{*}\right]+k_{\mathrm{trs}}[\mathrm{S}]\left[\mathrm{P}^{*}\right]
\end{aligned}
$$

where

$$
\begin{aligned}
k_{\mathrm{tc}}= & \text { termination constant for combination, } \\
k_{\mathrm{td}}= & \text { termination constant for } \\
& \text { disproportionation, } \\
k_{\mathrm{trm}}= & \text { transfer constant to monomer, }
\end{aligned}
$$

and

$$
k_{\mathrm{trs}}=\text { transfer constant to solvent. }
$$

The molecular weight distributions obtained in ECP under conditions of ideal mixing (i.e., no concentration gradients) are similar to those of nonECP processes in which the initiation rate is of the same form.

According to the steady-state assumption, the total concentration of all active sites $\left(P^{*}\right)$ remains constant during essentially the entire duration of the polymerization, so that

$$
R_{\mathrm{i}}=R_{\mathrm{t}}
$$

and the kinetic chain length,

$$
\nu=\frac{R_{\mathrm{p}}}{R_{\mathrm{i}}}
$$

The values of $[\mathrm{M}],\left[\mathrm{P}^{*}\right]$, and $[\mathrm{I}]$ in the latter equations for a particular ECP system depend on: (a) the types of initiation, termination, and transfer mechanisms; (b) the relative speeds of the various kinetic steps in the ECP process; (c) duration of the polymerization; and (d) distribution of the reacting species through the reaction space in the system. The latter is determined by the efficiency of mixing and the ease of diffusional processes in the reaction space.

Analyses of ECP processes support the following assumptions:

(i) Diffusion of all active species such as initiator radicals formed at the electrode is rapid with respect to the reaction rates of all the various mechanistic steps involved in the ECP. Also, mixing is efficient enough so that the concentration of each reactive species is uniform throughout the polymerization volume. Consequently, the diffusion phenomenon is not a significant factor and may be neglected.

(ii) The reaction rate constants are independent of polymer size.

(iii) Electrodes are not coated with resultant polymers.

\section{Free-Radical ECP}

To illustrate the analysis and behaviour of ECP processes, we shall discuss the case of a free-radical polymerization mechanism in which termination is of the second order (combination and/or disproportionation), and initiation is the result of a noncatalytic additive generated electrochemically and no chain transfer occurs.

For any combination of current-time in this system, one has

$$
\begin{aligned}
& R_{\mathrm{i}}=k_{\mathrm{i}}[\mathrm{M}]\left[\mathrm{I}^{\cdot}\right] \\
& R_{\mathrm{p}}=k_{\mathrm{p}}[\mathrm{M}]\left[\mathrm{P}^{\prime}\right] \\
& R_{\mathrm{t}}=k_{\mathrm{t}}\left[\mathrm{P}^{\cdot}\right]^{2}
\end{aligned}
$$

where

$$
k_{\mathrm{t}}=k_{\mathrm{tc}}+k_{\mathrm{td}},
$$

and the concentrations are related by 


$$
\begin{aligned}
& -\frac{\mathrm{d}[\mathrm{M}]}{\mathrm{d} t}=k_{\mathrm{i}}[\mathrm{M}]\left[\mathrm{I}^{\cdot}\right]+k_{\mathrm{p}}[\mathrm{M}]\left[\mathrm{P}^{\cdot}\right] \\
& \frac{\mathrm{d}\left[\mathrm{I}^{\cdot}\right]}{\mathrm{d} t}=\frac{i}{F V}-k_{\mathrm{i}}[\mathrm{M}]\left[\mathrm{I}^{\cdot}\right]
\end{aligned}
$$

and

$$
\frac{\mathrm{d}\left[\mathrm{P}^{`}\right]}{\mathrm{d} t}=k_{\mathrm{i}}[\mathrm{M}]\left[\mathrm{I}^{\bullet}\right]-k_{\mathrm{t}}\left[\mathrm{P}^{\cdot}\right]^{2}
$$

where

$i=$ constant current in amperes,

$V=$ volume of the solution,

$F=$ Faraday constant,

and

$i / F V=$ represents the quantity of the current in Faraday per unit volume required in the electrode process. These relations are not much different from those of a similar homogeneous chemically-initiated polymerization, ${ }^{7}$ the notable exception being the electrolytic current term in eq 11 . In eq 11 , it is assumed that only one electron is required to activate the non-catalytic initiator, $I$, to the free radical species, $I^{\circ}$. For example, this is true in the case of the electrochemical oxidation of carboxylate anions in the Kolbe synthesis of free radicals ${ }^{8-9}$ :

$$
\begin{aligned}
& \mathrm{R}-\mathrm{CO}_{2}^{-} \longrightarrow \mathrm{e}^{-} \text {(anode) }+\mathrm{RCO}_{2}^{-} \\
& \mathrm{RCO}_{2}^{-} \longrightarrow \mathrm{R}^{-}+\mathrm{CO}_{2}
\end{aligned}
$$

For a particular instance of constant current and steady-state, eq 11 gives

$$
\left[\mathrm{I}^{\cdot}\right]=\frac{i}{k_{\mathrm{i}} F V[\mathrm{M}]}
$$

and from eq 5, 7 and 9,

$$
\left[\mathrm{P}^{\prime}\right]=\left(\frac{i}{k_{\mathrm{t}} F V}\right)^{1 / 2}
$$

Equation 10 then gives

$$
-\frac{\mathrm{d}[\mathrm{M}]}{\mathrm{d} t}=\frac{i}{F V}+k_{\mathrm{p}}[\mathrm{M}]\left(\frac{i}{k_{\mathrm{t}} F V}\right)^{1 / 2}
$$

and on integration,

$[\mathrm{M}]=\left\{[\mathrm{M}]_{0}+\frac{k_{\mathrm{t}}^{1 / 2}}{k_{\mathrm{p}}}\left(\frac{i}{F V}\right)^{1 / 2}\right\}$

$$
\times \exp \left[-\frac{k_{\mathrm{p}}}{k_{\mathrm{t}}^{1 / 2}}\left(\frac{i}{F V}\right)^{1 / 2} t\right]-\frac{k_{\mathrm{t}}^{1 / 2}}{k_{\mathrm{p}}}\left(\frac{i}{F V}\right)^{1 / 2}
$$

If

$$
\frac{k_{\mathrm{t}}^{1 / 2}}{k_{\mathrm{p}}}=k,
$$

and

$$
\phi=\frac{i}{F V}
$$

then,

$$
[\mathrm{M}]=\left\{\left[\mathrm{M}_{0}+k \phi^{1 / 2}\right\} \exp \left(-\frac{\phi^{1 / 2}}{k}\right) t-k \phi^{1 / 2}\right.
$$

This result may be simplified further whenever high molecular weight polymers are formed. In this case, the monomer consumed by the initiator is negligible, and eq 10 becomes

$$
-\frac{\mathrm{d}[\mathrm{M}]}{\mathrm{d} t}=k_{\mathrm{p}}[\mathrm{M}]\left[\mathrm{P}^{\prime}\right]
$$

Using eq 16 , this gives

$$
-\frac{\mathrm{d}[\mathrm{M}]}{\mathrm{d} t}=k_{\mathrm{p}}[\mathrm{M}]\left(\frac{i}{k_{\mathrm{t}} F V}\right)^{1 / 2}
$$

Therefore, the initial rate of polymerization is proportional to the half power of electrolysis current. Thus a plot of the rate of polymerization versus the half power of current will give a straight line. Indeed, the rate of ECP of methyl methacrylate has been found experimentally to the proportional to the half power of the current. ${ }^{10}$

Equation 20 on integration gives

$$
\ln \frac{[\mathrm{M}]}{[\mathrm{M}]_{0}}=\frac{k_{\mathrm{p}}}{k_{\mathrm{t}}^{1 / 2}}\left(\frac{i}{F V}\right)^{1 / 2} t
$$

Then the ratio $k_{\mathrm{p}} / k_{\mathrm{t}}^{1 / 2}$ is the slope of a plot of $\ln [\mathrm{M}] /[\mathrm{M}]_{0} v s .(i / F V)^{1 / 2} t$ at a constant current. At low conversion, $[\mathrm{M}]=[\mathrm{M}]_{0}=$ constant, the kinetic chain length from eq 8 and 9 is

$$
\bar{v}=\frac{R_{\mathrm{p}}}{R_{\mathrm{t}}}=\frac{k_{\mathrm{p}}[\mathrm{M}]_{0}\left[\mathrm{P}^{\cdot}\right]}{k_{\mathrm{t}}\left[\mathrm{P}^{\cdot}\right]^{2}}=\frac{k_{\mathrm{p}}[\mathrm{M}]_{0}}{k_{\mathrm{t}}\left[\mathrm{P}^{\cdot}\right]}
$$

Substituting the value of $\left[\mathrm{P}^{*}\right]$ from eq 16 we have

$$
\bar{v}=\frac{k_{\mathrm{p}}[\mathrm{M}]_{0}}{k_{\mathrm{t}}^{1 / 2}(i / F V)^{1 / 2}}
$$

At significant conversions [M] departs from $[\mathrm{M}]_{0}$, and $v$ at the end of a polymerization time $\tau$, is obtained by averaging eq 22 over $\tau$ : 


$$
\bar{v}=\frac{k_{\mathrm{p}}}{\tau k_{\mathrm{t}}{ }^{1 / 2}(i / F V)^{1 / 2}} \int_{0}^{\tau}[\mathrm{M}] \mathrm{d} \tau
$$

and using (18) gives

$$
\bar{v}=\frac{1}{\tau} \frac{[\mathrm{M}]_{0}}{i / F V}\left[1-\exp \left\{-\frac{k_{\mathrm{p}}}{k_{\mathrm{t}}^{1 / 2}}\left(\frac{i}{F V}\right)^{1 / 2} \tau\right\}\right]
$$

To determine at what time $v$ is longest, let

$$
\begin{gathered}
k=\frac{k_{\mathrm{p}}}{\left(k_{\mathrm{t}}\right)^{1 / 2}}\left(\frac{i}{F V}\right)^{1 / 2}, \quad \text { then } \\
\frac{(i / F V)}{[\mathrm{M}]_{0}} \cdot \frac{(\mathrm{d} \vec{v})}{\mathrm{d} \tau}=0=-\frac{1}{\tau^{2}}+\frac{k}{\tau} \mathrm{e}^{-k \tau}+\frac{1}{\tau^{2}} \mathrm{e}^{-k \tau}
\end{gathered}
$$

i.e., $0=-1+(k \tau+1) \mathrm{e}^{-k \tau}$ or, $\mathrm{e}^{k \tau}=k \tau+1$ hence, $\bar{v}$ is maximum when $\tau=0$ and has the value,

$$
\begin{array}{rlr}
(\vec{v})_{\max } & =\frac{[\mathrm{M}]_{0}}{(i / F V)} \cdot \frac{1}{\tau}\left[1-\mathrm{e}^{-k \tau}\right] & (\tau \rightarrow 0) \\
& =\frac{[\mathrm{M}]_{0}}{(i / F V)}\left(k \mathrm{e}^{-k \tau}\right) & (\tau \rightarrow 0) \\
& =\frac{[\mathrm{M}]_{0} k}{(i / F V)}=\frac{k_{\mathrm{p}}[\mathrm{M}]_{0}}{k_{\mathrm{t}}^{1 / 2}(i / F V)^{1 / 2}} &
\end{array}
$$

which is the same result as eq 22 , as one would intuitively expect. However, the kinetic chain lengths of polymers made before the steady state is attained are larger than this value since the rate of termination is slower.

The above results are similar to those found for free-radical polymerizations initiated chemically or photochemically. However, the outstanding features of ECP are:

a) The initiation rates can be varied, at will, simply by controlling the applied electrolysis current (as for example, in controlling the light intensity in photopolymerization).

b) The initiation may be achieved by more different initiators and mechanisms from those of photopolymerization, thereby making it possible to determine the absolute reaction rate of polymerization.

\section{Anionic ECP}

A second example of the ECP process is that of anionic polymerization. In addition to those already mentioned, the following assumptions are made:

a) The slowest step is the dimerization of monomeric radical anions. b) Radical-anions are not capable of adding to monomer.

c) Initiation is via direct electron transfer from cathode to monomer.

d) Termination is only by reaction with solvent or other agent $(Z)$ and their reaction products are not capable of initiating polymerization.

e) Consumption of monomer is primarily by polymer growth, i.e., high molecular weight polymers are produced.

f) Concentration of solvent or chain terminating agent is essentially constant.

g) Steady-state concentration of polymeric anions is achieved.

The reaction steps may then be written as

$$
\begin{aligned}
& \mathrm{M}+\mathrm{e}^{-} \stackrel{k_{\mathrm{i}}}{\longrightarrow} \mathrm{M}^{-} \quad \text { (initiation) } \\
& 2 \mathrm{M}^{-} \stackrel{k_{\mathrm{d}}}{\longrightarrow} \mathrm{M}_{2}{ }^{-2} \\
& \quad \text { (dimerization, slowest step) } \\
& \mathrm{M}_{n}^{-2}+\mathrm{M} \stackrel{k_{\mathrm{p}}}{\longrightarrow} \mathrm{M}_{n+1}^{-2}, n \geq 2 \\
& \quad \text { (dianion growth) } \\
& \mathrm{M}_{n}^{-2}+\mathrm{Z} \stackrel{k_{\mathrm{t}}}{\longrightarrow} \mathrm{M}_{n}^{-}+\mathrm{Z}^{-}, n \geq 2 \\
& \quad \text { (termination) } \\
& \mathrm{M}_{n}{ }^{-}+\mathrm{Z} \stackrel{k_{\mathrm{t}}}{\longrightarrow} \mathrm{M}_{n}+\mathrm{Z}^{-}, n \geq 1 \\
& \mathrm{M}_{n}{ }^{-}+\mathrm{M} \stackrel{k_{\mathrm{p}}}{\longrightarrow} \mathrm{M}_{n+1}^{-1}, n \geq 1 \\
& \text { (monoanion growth) }
\end{aligned}
$$

By defining

$$
\left[\mathrm{P}^{-}\right]=\sum_{1}^{\infty}\left[2\left[\mathrm{M}_{n}^{-2}\right]+\left[\mathrm{M}_{n}^{-1}\right]\right]-\left[\mathrm{M}^{\top}\right],
$$

the rates of initiation, growth, and termination at steady-state, respectively, are

$$
\begin{aligned}
& R_{\mathrm{i}}=k_{\mathrm{d}}\left[\mathrm{M}^{-}\right]^{2} \\
& R_{\mathrm{p}}=k_{\mathrm{p}}[\mathrm{M}]\left[\mathrm{P}^{-}\right] \\
& R_{\mathrm{t}}=k_{\mathrm{t}}[\mathrm{Z}]\left[\mathrm{P}^{-}\right]
\end{aligned}
$$

consequently, the initial instantaneous kinetic chain length is

$$
v=\frac{R_{\mathrm{p}}}{R_{\mathrm{t}}}=\frac{k_{\mathrm{p}}[\mathrm{M}]}{k_{\mathrm{t}}[\mathrm{Z}]}
$$

Equation 29 holds only insofar as [M] and [Z] are constant; otherwise, $v$ must be averaged over the time of polymerization.

Since in this case the initiation rate is proportional to the constant current, the comments 
concerning $v$ at higher conversions in the freeradical section of this paper also hold here. The previous derivations yield ratios of rate constants only. This situation is analogous to that of chemical initiation. Determination of individual reaction rate constants requires some other methods. For example, molecular weight distribution, transient response to time-varying current, or a pulsed-current analog of the photopolymerization method for determining the life-time of intermediates. ${ }^{11}$ The above analysis may be summarized by the following equations, which govern the time dependence of anionic ECP:

$$
\frac{\mathrm{d}\left[\mathrm{M}^{-}\right]}{\mathrm{d} t}=\frac{i}{F V}-2 k_{\mathrm{d}}\left[\mathrm{M}^{-}\right]^{2}=0
$$

therefore,

$$
\left[\mathrm{M}^{\top}\right]=\left(\frac{i}{2 k_{\mathrm{d}} F V}\right)^{1 / 2}
$$

and

$$
\frac{\mathrm{d}\left[\mathrm{P}^{-}\right]}{\mathrm{d} t}=k_{\mathrm{d}}\left[\mathrm{M}^{-}\right]^{2}-k_{\mathrm{t}}[\mathrm{Z}]_{0}\left[\mathrm{P}^{-}\right]=0
$$

Therefore,

and

$$
\left[\mathrm{P}^{-}\right]=\frac{k_{\mathrm{d}}\left[\mathrm{M}^{-}\right]^{2}}{k_{\mathrm{t}}[\mathrm{Z}]_{0}}=\frac{i}{2 k_{\mathrm{t}} F V[\mathrm{Z}]_{0}}
$$

$$
\begin{gathered}
-\frac{\mathrm{d}[\mathrm{M}]}{\mathrm{d} t}=\frac{i}{F V}+k_{\mathrm{p}}[\mathrm{M}]\left[\mathrm{P}^{-}\right] \cong k_{\mathrm{p}}[\mathrm{M}]\left[\mathrm{P}^{-}\right] \\
=\frac{k_{\mathrm{p}}[\mathrm{M}]}{k_{\mathrm{t}}[\mathrm{Z}]_{0}}\left(\frac{i}{2 F V}\right)
\end{gathered}
$$

Therefore, for low conversions (i.e., initial stages), the rate of polymerization may be evaluated from eq 35 .

\section{Some Limiting Cases of Anionic ECP}

The relative importance of the various mechanistic steps in the kinetics of electrochemical initiation, by direct electron transfer between monomer and electrode (cathod or anode), may be determined by analysis of current-voltage-concentration relationships. For example, consider the electrochemical reduction of an unsaturated (i.e., containing $a \pi$ or double bond) monomer:

$$
\mathrm{M}+\mathrm{e}^{-} \underset{k_{\mathrm{bh}}}{\stackrel{k_{\mathrm{fh}}}{\rightleftarrows}} \mathrm{M}^{-}
$$

$$
\begin{aligned}
& 2 \mathrm{M}^{-} \stackrel{k_{\mathrm{d}}}{\longrightarrow} \mathrm{M}_{2}^{-2} \\
& \mathrm{M}_{2}^{-2}+\mathrm{M} \stackrel{k_{\mathrm{p}}}{\longrightarrow} \mathrm{M}_{n+1}^{-2}
\end{aligned}
$$

For simplicity, assume that the coupling of $\mathrm{M}^{-}$ occurs only on the electrode surface and that polymerization occurs only in the solution. The latter implies that diffusion of $\mathrm{M}_{2}{ }^{-2}$ away from the electrode is rapid relative to the addition of $M$ to $\mathrm{M}_{2}{ }^{-2}$, a condition which may be achieved in certain real systems. The reaction under consideration is similar to that used for studying the electrochemical reduction of non-polymerizing species. ${ }^{12,13}$

At steady-state, the rate of electron transfer equals the rate of $\mathrm{M}^{-}$coupling. Since coupling occurs only on the electrode surface, the surface concentration of $\mathrm{M}^{-}$is represented by the fraction of surface covered by $\mathrm{M}^{-}$, viz. $\theta .^{*}$ Thus,

$$
\begin{aligned}
\frac{\mathrm{d} \theta}{\mathrm{d} t} & =0=k_{\mathrm{fh}}(1-\theta)[\mathrm{M}]-k_{\mathrm{bh}} \theta-2 k_{\mathrm{d}} \theta^{2} \\
& =k_{\mathrm{fh}}[\mathrm{M}]-\theta\left(k_{\mathrm{fh}}[\mathrm{M}]+k_{\mathrm{bh}}\right)-2 k_{\mathrm{d}} \theta^{2}
\end{aligned}
$$

which gives

$$
\begin{aligned}
\theta= & \frac{1}{4 k_{\mathrm{d}}}\left\{-\left(k_{\mathrm{bh}}+k_{\mathrm{fh}}[\mathrm{M}]\right) \pm\left[\left(k_{\mathrm{bh}}+k_{\mathrm{fh}}[\mathrm{M}]\right)^{2}\right.\right. \\
& \left.\left.+8 k_{\mathrm{fh}}[\mathrm{M}] k_{\mathrm{d}}\right]^{1 / 2}\right\}
\end{aligned}
$$

From this, the following limiting cases arise:

(A) For slow electron transfer $\left(k_{\mathrm{d}} \gg k_{\mathrm{fh}}, k_{\mathrm{bh}}\right)$

$$
\theta \simeq\left(\frac{k_{\mathrm{fh}}[\mathrm{M}]}{2 k_{\mathrm{d}}}\right)^{1 / 2}
$$

and the rate of electron transfer equals the rate of $\mathrm{M}^{-}$coupling, so that the cathodic current is

$$
\begin{aligned}
i_{\mathrm{c}} & =2 F k_{\mathrm{d}} \theta^{2} \\
& =2 F k_{\mathrm{d}} \frac{k_{\mathrm{fh}}[\mathrm{M}]}{2 k_{\mathrm{d}}}=F k_{\mathrm{fh}}[\mathrm{M}]
\end{aligned}
$$

$k_{\mathrm{fh}}$ is given by ${ }^{12,13}$

$$
k_{\mathrm{fh}}=k_{\mathrm{fh}}^{0} \exp \left(\frac{-n \alpha F E}{R T}\right)
$$

* Notes: (i) This analysis is valid only if no polymer deposits on the electrode and if no other species compete for adsorption on the electrode.

(ii) The ratio, $k_{\mathrm{bh}}^{0} / k_{\mathrm{fh}}^{0}$, is constant as long as the electrode surface is chemically and physically stable. 
where $k_{\mathrm{fh}}$ is the rate constant of rate determining step, $n$ is the number of electrons in the rate determining step, and $E$ is the electrode potential (referred to normal hydrogen electrode). The fraction $\alpha E$ of this potential favours the cathodic reaction and fraction $(1-\alpha) E$ favours the anodic reaction. The parameter $\alpha$ is the transfer coefficient for the cathodic process, the range of which is $0<\alpha<1 .{ }^{14}$ For simplicity, we shall assume a symmetrical energy barrier for the electron transfer, i.e.; $\alpha=0.5$.

In this case, $n=1$, and taking $\alpha=0.5$,

$$
i_{\mathrm{c}}=F k_{\mathrm{fh}}^{0}[\mathrm{M}] \exp \left(\frac{-F E}{2 R T}\right)
$$

and

$$
\frac{\mathrm{d} \ln i_{\mathrm{c}}}{\mathrm{d} E}=-\frac{F}{2 R T}
$$

and

$$
\frac{\mathrm{d} \theta}{\mathrm{d} E}=-\frac{F \theta}{4 R T}
$$

This situation is favoured by low monomer concentration, [M].

(B) For slow surface coupling relative to electron transfer, $k_{\mathrm{d}}$ is negligible, and hence

$$
\theta \simeq \frac{k_{\mathrm{fh}}[\mathrm{M}]}{k_{\mathrm{fh}}[\mathrm{M}]+k_{\mathrm{bh}}}
$$

and for small cathodic potentials and low [M],

$$
\theta \simeq \frac{k_{\mathrm{fh}}[\mathrm{M}]}{k_{\mathrm{bh}}}
$$

therefore,

$$
\begin{aligned}
& i_{\mathrm{c}}=2 F k_{\mathrm{d}} \theta^{2}=2 F k_{\mathrm{d}}\left(\frac{k_{\mathrm{fh}}[\mathrm{M}]}{k_{\mathrm{bh}}}\right)^{2} \\
& =2 F k_{\mathrm{d}}\left(\frac{k_{\mathrm{fh}}^{0}[\mathrm{M}]}{k_{\mathrm{bh}}^{0}}\right)^{2} \exp \left(\frac{-2 F E}{R T}\right) \\
& \quad(\text { as } n=1, \alpha=0.5)
\end{aligned}
$$

then

$$
\frac{\mathrm{d} \ln i_{\mathrm{c}}}{\mathrm{d} E}=-\frac{2 F}{R T}
$$

From eq 48,

$$
\frac{\mathrm{d} \theta}{\mathrm{d} E}=-\frac{F \theta^{2} k_{\mathrm{bh}}^{0}}{R T k_{\mathrm{fh}}[\mathrm{M}]} \exp \left(\frac{F E}{R T}\right)
$$

However, for more cathodic potentials and high $[\mathrm{M}], \theta \simeq 1.0$ and

$$
i_{\mathrm{c}}=2 F k_{\mathrm{d}}=\left(i_{\mathrm{c}}\right)_{\mathrm{L}}=\text { limiting current }
$$

then,

$$
\frac{\mathrm{d} \ln i_{\mathrm{c}}}{\mathrm{d} E}=0
$$

and,

$$
\frac{\mathrm{d} \theta}{\mathrm{d} E}=0
$$

From the results (A) and (B), above, we conclude that these distinct limiting cases can be distinguished by examining $i-E$ curves under appropriate conditions, when there is dimerization of the. intermediate radical-anion $\mathrm{M}^{-}$. However, it is conceivable for some $\pi$ bonded monomers that $\mathrm{M}^{-}$ prefers to add another neutral monomer anionically before, or concerted with, rapid acceptance of a second electron from the electrodes:

$$
\begin{aligned}
& \mathrm{M}+\mathrm{e}^{-} \underset{k_{\mathrm{bh}}}{\stackrel{k_{\mathrm{fh}}}{\rightleftarrows}} \mathrm{M}^{-} \\
& \mathrm{M}^{-}+\mathrm{M} \stackrel{k_{\mathrm{a}}}{\longrightarrow} \cdot \mathrm{M}-\mathrm{M}^{-} \\
& \mathrm{M}-\mathrm{M}^{-}+\mathrm{e}^{-} \stackrel{k_{\mathrm{fh}}^{\prime}}{\longrightarrow}-\mathrm{M}-\mathrm{M}^{-}
\end{aligned}
$$

Regardless of which of these steps is rate controlling, the steady-state surface coverage by $\mathrm{M}^{-}$is

$$
\theta=\frac{k_{\mathrm{fh}}[\mathrm{M}]}{\left(k_{\mathrm{fh}}+k_{\mathrm{a}}\right)[\mathrm{M}]+k_{\mathrm{bh}}}
$$

Now we consider the following limiting cases of this scheme.

(C) If the first electron transfer (56) is much slower than monomer addition to radical anion (57), then for cathodic potentials, $\theta$ is small and,

and

$$
\theta \simeq \frac{k_{\mathrm{fh}}}{k_{\mathrm{a}}}
$$

$$
\begin{aligned}
i_{\mathrm{c}} & =2 F k_{\mathrm{a}}[\mathrm{M}] \theta \\
& =2 F k_{\mathrm{fh}}^{0}[\mathrm{M}] \exp \left(\frac{-F E}{2 R T}\right)
\end{aligned}
$$

then,

$$
\frac{\mathrm{d} \ln i_{\mathrm{c}}}{\mathrm{d} E}=-\frac{F}{2 R T}
$$


and

$$
\frac{\mathrm{d} \theta}{\mathrm{d} E}=-\frac{F \theta}{2 R T}
$$

(D) If the addition of $M$ to $M^{-}$is the slowest step, two distinct cases arise dependent on the ratio $k_{\mathrm{fh}}[\mathrm{M}] / k_{\mathrm{bh}}$, which determines $\theta$. For $k_{\mathrm{fh}}[\mathrm{M}] \ll k_{\mathrm{bh}}$,

$$
\theta \simeq \frac{k_{\mathrm{fh}}[\mathrm{M}]}{k_{\mathrm{bh}}}
$$

hence,

then,

$$
\begin{aligned}
i_{\mathrm{c}} & \simeq 2 F k_{\mathrm{a}}[\mathrm{M}] \theta \\
& =2 F[\mathrm{M}]^{2} \frac{k_{\mathrm{a}} k_{\mathrm{fh}}^{0}}{k_{\mathrm{bh}}^{0}} \exp \left(\frac{-F E}{R T}\right)
\end{aligned}
$$

$$
\begin{gathered}
\frac{\mathrm{d} \ln i_{\mathrm{c}}}{\mathrm{d} E}=-\frac{F}{R T} \\
\frac{\mathrm{d} \theta}{\mathrm{d} E}=-\frac{F \theta}{R T}
\end{gathered}
$$

However, for $k_{\mathrm{fh}}[\mathrm{M}] \gg k_{\mathrm{bh}}, \theta \simeq 1.0$ so that,

$$
i_{\mathrm{c}} \simeq 2 F k_{\mathrm{a}}[\mathrm{M}]
$$

then,

$$
\frac{\mathrm{d} \ln i_{\mathrm{c}}}{\mathrm{d} E}=0
$$

and

$$
\frac{\mathrm{d} \theta}{\mathrm{d} E}=0
$$

(E) If the second electron transfer (58) is slowest, all preceding reactions $(56,57)$ will be nearly at equilibrium giving rise to different forms of the Tafel relationships. Since in practice, this electron transfer is very fast compared to the other steps, there is no need to work out these cases. From the derived Tafel relationships for current and electrode potential, various limiting schemes can be distinguished theoretically. The results so obtained are presented in Table I. It is seen from this table that $i-$ $E$ relationships alone do not distinguish between the electron transfer-limited dimerization (A) from that of monomer addition (C). However, if $\theta-E$ relationships can be determined experimentally, then these two cases can be distinguished. From a measurement of surface tension at the electrodesolution interface, determination of double layer capacitance dependence on frequency and analyses

\begin{tabular}{|c|c|c|c|c|c|c|}
\hline Class & Mechanism & Slowest step & $\frac{\mathrm{d} \ln i_{\mathrm{c}}}{\mathrm{d} E}$ & & $\frac{\mathrm{d} \theta}{\mathrm{d} E}$ & \\
\hline (A) & $\begin{array}{l}\text { Radical-anion } \\
\text { dimerization }\end{array}$ & $\begin{array}{l}\text { Electron } \\
\text { transfer }\end{array}$ & $-\frac{F}{2 R T}$ & $-\frac{F \theta}{4 R T}$ & & \\
\hline \multirow[t]{2}{*}{ (B) } & \multirow[t]{2}{*}{$\begin{array}{l}\text { Radical-anion } \\
\text { dimerization }\end{array}$} & $\begin{array}{l}\text { Dimerization } \\
\text { (1) Small potential and }[\mathrm{M}] \\
(\text { low } \theta)\end{array}$ & $-\frac{2 F}{R T}$ & \multicolumn{2}{|c|}{$\frac{F \theta^{2} k_{\mathrm{bh}}^{0}}{R T k_{\mathrm{fh}}[\mathrm{M}]}$} & $\left(\frac{F E}{R T}\right)$ \\
\hline & & $\begin{array}{l}\text { (2) Large potential and [M] } \\
(\theta \rightarrow 1.0)\end{array}$ & 0 & & 0 & \\
\hline (C) & $\begin{array}{l}\text { Radical-anion } \\
\text { monomer addition }\end{array}$ & $\begin{array}{l}\text { First electron } \\
\text { transfer }\end{array}$ & $-\frac{F}{2 R T}$ & $-\frac{F \theta}{2 R T}$ & & \\
\hline (D) & $\begin{array}{l}\text { Radical-anion } \\
\text { monomer addition }\end{array}$ & $\begin{array}{l}\text { Monomer } \\
\text { addition } \\
\text { (1) low } \theta \\
\text { (2) } \theta \rightarrow 1.0\end{array}$ & $\begin{array}{r}-\frac{F}{R T} \\
0\end{array}$ & $-\frac{F \theta}{R T}$ & 0 & \\
\hline
\end{tabular}
of electrochemical charging and decay curves, $\theta-E$ relationship is obtained.

\section{Effects of Diffusion}

In deriving the relationship in the preceding sections it was assumed that the rate of transport of active species between the electrode surface and the reaction mixture was much faster than that of

Table I. Characteristics of proposed direct initiation electrode reactions 
polymerization processes $^{15}$ :

$$
\frac{D}{\bar{\delta}}\left[\mathrm{P}^{*}\right] \gg k_{\mathrm{p}}[\mathrm{M}]\left[\mathrm{P}^{*}\right]
$$

where $D=$ the coefficient of diffusion and $\bar{\delta}=$ the average diffusion layer thickness. For a flat electrode in turbulent fluid, the average $\delta$ across the electrode may be given by ${ }^{16}$

$$
\bar{\delta}=l \operatorname{Re}^{-0.9} \operatorname{Pr}^{-0.33}
$$

where

$l=$ the coordinate in the direction of current flow measured from the edge of the electrode,

$R e=$ Reynold's number,

and

$P r=$ Prandt's number.

This expression shows that $\bar{\delta}$ decreases with the increasing $R e$ values. Therefore, in order to minimize diffusion effects, vigorous agitation is essential. Under such conditions, our derived formulations are applicable.

\section{REFERENCES}

1. B. L. Funt, Macromol. Rev., 1, 35 (1967).
2. N. Yamazaki, Adv. Polym. Sci., 6, 377 (1969).

3. J. W. Breitenbach, O. F. Olaj, and F. Somer, $A d v$. Polym. Sci., 9, 47 (1972).

4. G. S. Shapoval and A. V. Gorodyskii, Russ. Chem. Rev., 42, 370 (1973).

5. G. Parravano, "Organic Electrochemistry," M. M. Baizer Ed., Marcel Dekker, New York, 1973, p 947.

6. K. J. Vetter, "Electrochemical Kinetics," Academic Press, New York-London, 1967.

7. G. E. Ham, "Vinyl Polymerization," G. E. Ham Ed., Marcel Dekker, New York, 1967, Part 1, p 10.

8. M. R. Rifi and F. H. Covitz, "Intoduction to Organic Electrochemistry," Marcel Dekker, New York, 1974, p 256.

9. A. K. Vigh and B. E. Conway, Chem. Rev., 67, 623 (1967).

10. B. L. Funt and S. N. Bhadani, J. Polym. Sci., A, 3, 4191 (1965).

11. P. J. Flory, "Principles of Polymer Chemistry," Cornell Univ. Press, Ithaca, New York, 1953, p 148.

12. P. Delahay, "New Instrumental Methods in Electrochemistry," Interscience Publishers, Inc., New York, 1966, p 32.

13. S. Glasstons, K. J. Laidler, and H. Eyring, "The Theory of Rate Processes," McGraw Hill, New York, 1941, p 575.

14. ref $6, \mathrm{p} 117$.

15. ref $13, \mathrm{p} 578$.

16. $\operatorname{ref} 6, \mathrm{p} 191$. 